\title{
Expanding the Sense of Touch Outside the Body
}

\author{
Christopher C. Berger ${ }^{*}$ \\ California Institute of Technology \\ Pasadena, CA, USA \\ cberger@caltech.edu
}

\author{
Mar Gonzalez-Franco \\ Microsoft Research \\ Redmond, USA \\ margon@microsoft.com
}

\begin{abstract}
Under normal circumstances, our sense of touch is limited to our body. Recent evidence suggests, however, that our perception of touch can also be expanded to objects we are holding when certain tactile illusions are elicited by delivering vibrotactile stimuli in a particular manner. Here, we examined whether an extra-corporeal illusory sense of touch could be elicited using vibrotactile stimuli delivered via two independent handheld controllers while in virtual reality. Our results suggest that under the right conditions, one's sense of touch in space can be extended outside the body, and even into the empty space that surrounds us. Specifically, we show, in virtual reality, that one's sense of touch can be extended to a virtual stick one is holding, and also into the empty space between one's hands. These findings provide a means with which to expand the sense of touch beyond the hands in VR systems using two independent controllers, and also have important implications for our understanding of the human representation of touch.
\end{abstract}

\section{CCS CONCEPTS}

- Human-centered computing $\rightarrow$ Virtual reality; Haptic devices; Empirical studies in $\mathrm{HCI}$;

\section{KEYWORDS}

Saltation, Cutaneous Rabbit Illusion, Tactile Funneling Illusion, Haptics, Multisensory Perception, Virtual Reality

\section{ACM Reference Format:}

Christopher C. Berger and Mar Gonzalez-Franco. 2018. Expanding the Sense of Touch Outside the Body. In SAP '18: ACM Symposium on Applied Perception 2018, August 10-11, 2018, Vancouver, BC, Canada. ACM, New York, NY, USA, 9 pages. https://doi.org/10.1145/3225153.3225172

\section{INTRODUCTION}

Our sense of touch is usually limited to our bodies. However, our perception of touch does not always correspond to the location of physical contact with the skin [Burton 1993]. One classic example, is the "cutaneous rabbit" illusion whereby a series of rapid taps delivered first at one location on the skin and then at another leads to the illusory perception that one was touched in the intervening

\footnotetext{
${ }^{*}$ This is the corresponding author

Permission to make digital or hard copies of all or part of this work for personal or classroom use is granted without fee provided that copies are not made or distributed for profit or commercial advantage and that copies bear this notice and the full citation on the first page. Copyrights for components of this work owned by others than ACM must be honored. Abstracting with credit is permitted. To copy otherwise, or republish, to post on servers or to redistribute to lists, requires prior specific permission and/or a fee. Request permissions from permissions@acm.org.

SAP '18, August 10-11, 2018, Vancouver, BC, Canada

(C) 2018 Association for Computing Machinery.

ACM ISBN 978-1-4503-5894-1/18/08 . \$15.00

https://doi.org/10.1145/3225153.3225172
}

space on the skin [Geldard and Sherrick 1972]. This illusion is called the "cutaneous rabbit" illusion because it simulates the experience of a rabbit hopping between the actual sites of stimulation. Similarly, an illusory sense of touch can be felt between two disparate points as a result of the 'funneling action' of the nervous system, whereby simultaneous vibrotactile stimulation at two locations on the skin leads to an illusory perception that the source of the touch is in the intervening space [v. Békésy 1958]. In the tactile funneling illusion, the perceived location of the vibrotactile stimuli can be changed by manipulating the amplitude of the two sources of vibrotactile stimuli of the same waveform and frequency.

Tactile illusions like the cutaneous rabbit (CRI) and tactile funneling illusion, have stirred a debate among psychologists and neuroscientists about how the brain represents the sense of touch. On one side were those who argue that the sense of touch (illusory or otherwise) is the product of activation of location-specific receptive fields in the primary somatosensory cortex [Flach and Haggard 2006; Geldard and Sherrick 1972]. On the other side are those that argue that receptive-field mapping in the somatosensory cortex is not sufficient to explain the influence of cognitive factors on our perception of touch, such as selective attention and the across-arm CRI [Eimer et al. 2005; Kilgard and Merzenich 1995]. In the case of the CRI, the debate was believed to be settled, when neuroimaging evidence revealed that the illusory sense of touch was correlated with activation at the site of the primary somatosensory cortex that represents the portion of the skin where the illusory perception of touch was felt [Blankenburg et al. 2006]. Thus, it was confirmed that our sense of touch, even when illusory, is represented by the primary somatosensory cortex and in a somatotopically organized manner.

Subsequent work by Miyazaki, Hirashima, \& Nozaki [Miyazaki et al. 2010], however, revealed that the CRI could also be extended to an object one is holding. Miyazaki et al. [Miyazaki et al. 2010] found that if vibrotactile stimuli are delivered in rapid succession first to one index finger, then on the next while one is holding an object between their fingers an illusory 'out-of-the-body cutaneous rabbit illusion' is experienced in the intervening space between the fingers on the object they were holding. These findings were also consistent with earlier findings on the tactile funneling illusion by Chen, Friedman, \& Roe [Chen et al. 2003] who found that simultaneous tactile stimulation to two fingertips elicited activation in a single focal point between the representation of each finger in the primary somatosensory cortex of squirrel monkeys. The amplitude of the activation in the merged focal site between the cortical representation for each finger was comparable to the amplitude of the activation when each finger was stimulated individually with the same intensity [Chen et al. 2003]. Together, these finding suggests that we can experience a sense of touch on objects that are not represented by the intrinsic samatotopic representation of the body, 
and more broadly, that our sense of touch is not necessarily limited to the surface of our skin.

Research and development of technological applications for delivering haptic experiences from vibrotactile actuators have begun to make use of the CRI and tactile funneling illusion in order to elicit robust tactile experiences using fewer points of contact for vibrotactile actuators [Hoggan et al. 2007; Richter et al. 2011; Schneider et al. 2015]. However, this line of work and research in this area has primarily focused tactile experiences in the absence of information from other sensory cues [Chen et al. 2003; Geldard and Sherrick 1972; Hoggan et al. 2007; Miyazaki et al. 2010; Richter et al. 2011; Schneider et al. 2015], and therefore, little is known about the influence of information from our other senses on these tactile illusions (cf. [Asai and Kanayama 2012; Lee et al. 2012; Pittera et al. 2017]. This poses a problem for potential real-world applications as well as our understanding of our sense of touch more generally, because, we live in a multisensory environment wherein the brain integrates all available information from our different senses in order to create a coherent perception of the world around us [Ghazanfar and Schroeder 2006; Stein and Stanford 2008]. Thus, in order to develop a comprehensive understanding of tactile illusions like the CRI and tactile funneling illusion, and orient developers towards fruitful applications, it is necessary to examine these illusions in a multisensory context. Moreover, given the dominant role of vision in our everyday lives, it is important to know whether theses tactile illusions can even occur in contexts in which users have their eyes open; and if so, how visual information interacts with these illusory tactile percepts.

Recent work in this area has explored whether the out-of-thebody CRI and tactile funneling illusion can be experienced when participants receive vibrotactile stimuli delivered to their index fingers with their eyes open [Lee et al. 2012]. Results from this work suggest that both the CRI and tactile funneling illusion can be experienced when the eyes are open when visual information (e.g., a 'virtual' ruler rendered between the fingers viewed on 2D display) is available to 'bind' the tactile stimuli in space [Lee et al. 2012]. However, one limitation of this work is that the visual information displayed to the participants was viewed on a $2 \mathrm{D}$ display in front of the participants rather than from the natural first-person perspective. For this reason, these findings may merely reflect the shift in executive attention away from participants' real hands, rather than a genuine tactile experience. Thus, it unknown whether the out-of-the-body illusory sense of touch can be experienced from a first-person perspective, and if so, how visual information interacts with the out-of-the-body illusory sense of touch. Here, in three separate experiments, we made use of the CRI and tactile funneling illusion in an immersive virtual reality (VR) setup to explore whether one's sense of touch could be expanded to virtual objects or even empty space when receiving visuotactile stimuli from a first person perspective while one's eyes are open.

\section{EXPERIMENT 1}

In the first experiment, we sought to examine whether an illusory out-of-the-body sense of touch could be elicited using the 'cutaneous rabbit illusion' (CRI) technique of vibrotactile stimulation in an immersive VR setting. This experiment consisted of four experimental conditions that were designed to probe the relative importance of holding a virtual object between the hands and the inter-stimulus timing intervals (ISIs) of the successive vibrotactile stimuli. In one condition, three vibrotactile stimuli were presented sequentially-the first two to one hand and the third to the other hand using ISIs known to elicit the CRI [Geldard and Sherrick 1972; Miyazaki et al. 2010] while the participant was holding a virtual stick rendered between their hands and a white sphere was presented simultaneously with each vibrotactile stimulus (CRI ISIs + Stick + White Spheres condition). The first sphere was presented in the same location as the first vibrotactile stimulus, the second sphere was presented in the intended illusory location (i.e., the mid-point between the left and right hands) and the third was presented at the same location as the third vibrotactile stimulus. In the other three conditions, we examined whether any observed out-of-the-body illusory sense of touch would be experienced if no stick was rendered between the participants hands (CRI ISIs + No Stick + White Spheres condition) or if we altered ISI between the vibrotactile and visual stimuli when a stick was present (Non-CRI + Stick + White Spheres condition), and when no stick was present (Non-CRI ISIs + No Stick + White Spheres condition). The altered ISIs (i.e., non-CRI ISIs) were chosen in order to maintain the same overall duration as the CRI ISIs, and because the spatiotemporal relationship between vibrotactile stimuli in the non-CRI ISI conditions was in line with normal haptic perception (i.e. tactile stimuli occurring close in time usually occur close in space, whereas more spatially disparate tactile stimuli occur across a larger temporal interval), no illusory sense of touch for the second vibrotactile pulse was anticipated. Thus, the non-CRI conditions served as a control for participant response bias. Additionally, previous research on multisensory perception and multisensory integration suggests that the brain integrates congruent sensory cues from different sensory modalities in order to form coherent, and sometimes enhanced, sensory percepts [Ghazanfar and Schroeder 2006; Stein and Stanford 2008]. Consistent with this line of research, findings from a study conducted by Asai \& Kanayama [Asai and Kanayama 2012] using the CRI revealed that the CRI was enhanced when visual cues congruent with the perceived illusion of touch were presented. Thus, here we hypothesized that vibrotactile stimuli delivered to the participants using CRI ISIs, together with the visual cues of the white spheres and the virtual stick would elicit a robust illusion of touch on the virtual stick in the space between the hands, and that this illusion would be diminished or absent when the stick was absent, or the non-CRI ISIs were used.

\subsection{Method}

2.1.1 Participants. Twenty participants participated in Experiment 1 (mean age $=33.9$ years, $\mathrm{SD}=9.03 ; 4$ females). All participants were recruited from within Microsoft Research, were healthy, reported no history of psychiatric illness or neurologic disorder, and reported no impairments of hearing or vision (or had corrected-tonormal vision).

2.1.2 Materials and Procedure. The participants received vibrotactile stimuli delivered in rapid succession to the ventral pads of the left and right index fingers from two independent handheld HTC Vive controllers while inside the virtual environment (see 

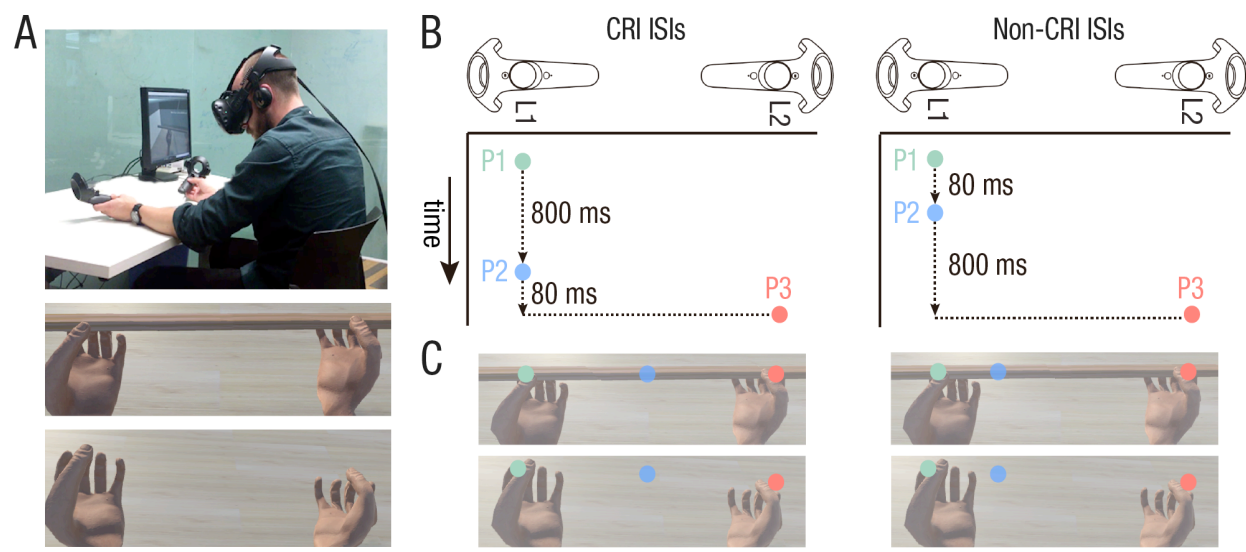

D

CRI ISls + Stick + White Spheres

Non-CRI ISIs + Stick + White Spheres
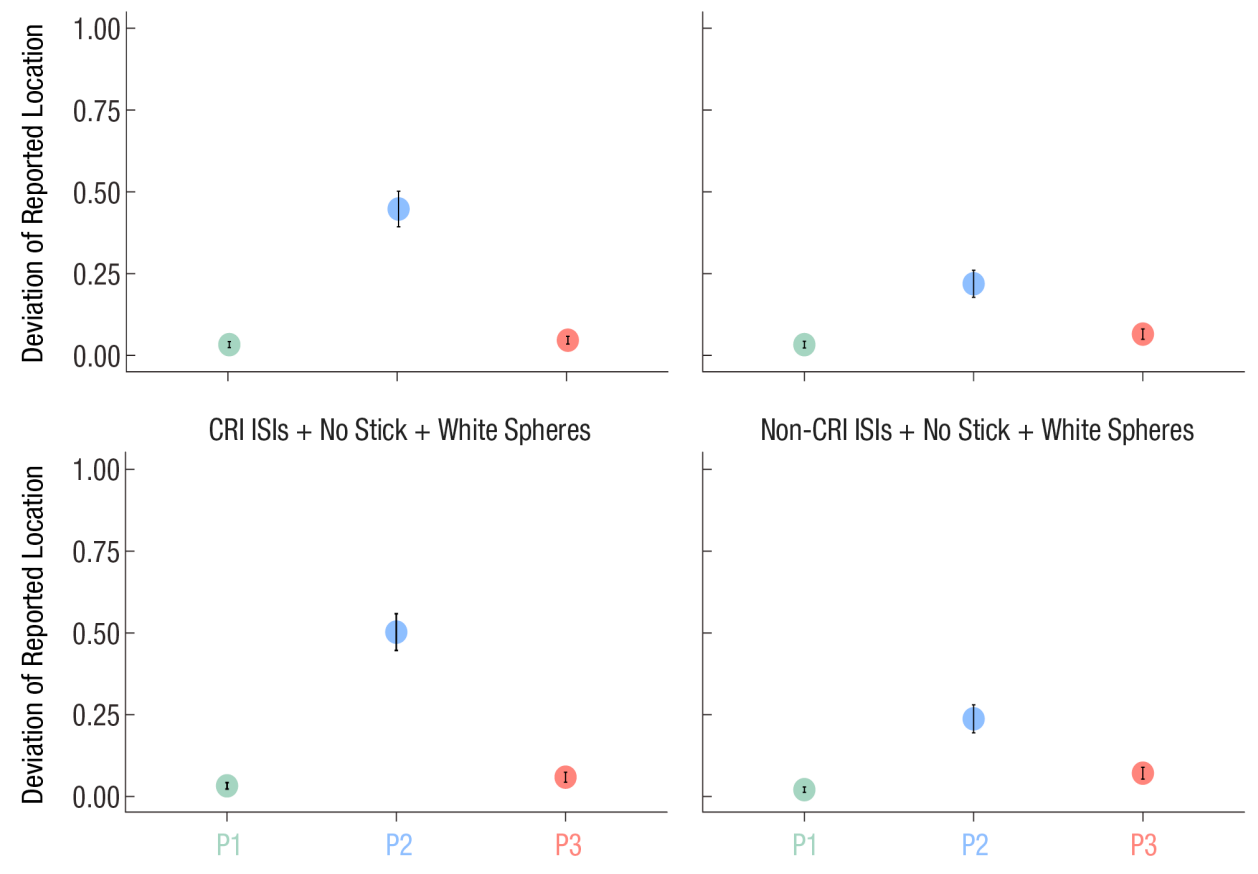

Figure 1: Using the Cutaneous Rabbit Illusion to create an Out-of-the-Body Touch Illusion. (A) The posture of the participants during the experiment (top), and the first person perspective of the hands as seen through he HMDs during the conditions when a virtual stick was rendered between the virtual hands (middle), and when it was not (bottom). (B) A schematic overview of the spatio-temporal relationship between each vibrotactile pulse (P1-P3) delivered via the left and right HTC Vive controllers. (C) Typical reported location for each Pulse (P1-P3) when the cutaneous rabbit ISIs were used both when the stick was present (top left) and when it was not present (bottom left), and when the non-cutaneous rabbit ISIs were used, both when the stick was present (top right) and not present (bottom right). (D) Mean deviation of the participants' reported location for each vibrotactile pulse in the conditions in which the cutaneous rabbit illusion ISIs were used and a stick was present (top left) or absent (bottom left), and when the non-cutaneous rabbit illusion ISIs were used and the stick was present (top right) or absent (bottom right). White spheres were presented simultaneously with each vibrotactile pulse. The second white sphere presented in a sequence was always presented in the intended illusory location (i.e., between the left and right hands). Error bars represent +/- 1 SEM. 
Table 1: Mean deviation comparisons for Experiment 1.

\begin{tabular}{llcccc}
\hline Condition and Comparison & $t$ & $\mathrm{df}$ & $\mathrm{p}$-value & $95 \% \mathrm{Cl}$ & Cohen's D \\
\hline Stick Present + Cutaneous Rabbit Timing & & & & & \\
P1 vs 0 & 3.50 & 19 & $.043^{*}$ & $.01-.05$ & 0.78 \\
P2 vs P1 & 7.57 & 19 & $<.001^{* * *}$ & $.29-.52$ & 1.69 \\
P2 vs P3 & 7.19 & 19 & $<.001^{* * *}$ & $.03-.52$ & 1.61 \\
P3 vs P1 & 1.20 & 19 & 1.00 & $-.01-.04$ & 0.25 \\
Stick Absent + Cutaneous Rabbit Timing & & & & & \\
P1 vs 0 & 3.30 & 19 & 0.067 & $.01-.05$ & 0.74 \\
P2 vs P1 & 8.54 & 19 & $<.001^{* * *}$ & $.35-.58$ & 1.91 \\
P2 vs P3 & 7.36 & 19 & $<.001^{* * *}$ & $.32-.57$ & 1.65 \\
P3 vs P1 & 1.63 & 19 & 1.00 & $-.01-.06$ & 0.36 \\
Stick Present + Non-Cutaneous Rabbit Timing & & & & & \\
P1 vs 0 & 3.21 & 19 & $.033^{*}$ & $.01-.05$ & 0.72 \\
P2 vs P1 & 4.64 & 19 & $.003^{* *}$ & $.10-.27$ & 1.03 \\
P2 vs P3 & 3.75 & 19 & $.025^{*}$ & $.07-.24$ & 0.84 \\
P3 vs P1 & 1.98 & 19 & 1.00 & $-.00-.07$ & 0.44 \\
Stick Absent + Non-Cutaneous Rabbit Timing & & & & & \\
P1 vs 0 & 2.70 & 19 & .256 & $.01-.04$ & 0.60 \\
P2 vs P1 & 5.43 & 19 & $<.001^{* * *}$ & $.13-.29$ & 1.22 \\
P2 vs P3 & 3.93 & 19 & $.016^{*}$ & $.08-.25$ & 0.88 \\
P3 vs P1 & 2.90 & 19 & .166 & $.01-.09$ & 0.65 \\
\hline Note: All p-values are bonferroni-corrected for multiple comparisons. ${ }^{*} p<.05, * * p<.01, * * * p<.001$. & &
\end{tabular}

Figure 1A). Each vibrotactile stimulus was $60 \mathrm{~ms}$ in duration at the maximum amplitude of the HTC Vive controllers. The temporal sequence of the three vibrotactile stimuli was consistent with previous studies on the cutaneous rabbit illusion [Asai and Kanayama 2012; Miyazaki et al. 2010; Warren et al. 2010]. The first pulse (P1) was first delivered at the first location (L1), followed by the second tap (P2) delivered again to the first location, and finally a third tap delivered to the second location (L2). In one condition, the interstimulus interval (ISI) between P1 and P2 at L1 was $800 \mathrm{~ms}$, and the ISI between $\mathrm{P} 2$ at $\mathrm{L} 1$ and $\mathrm{P} 3$ at $\mathrm{L} 2$ was $80 \mathrm{~ms}$. In the control condition, the ISI between P1 and P2 was $80 \mathrm{~ms}$, and the ISI between P2 and P3 was 800 ms (see Figure 1B). There were twenty trials per block, and 4 blocks in total. Each trial consisted of a vibrotactile stream followed by the participants' report of the perceived location of each tap, and the strength rating of each tap. A virtual stick was rendered between the hands of the virtual avatar in 2 of the 4 blocks of trials, and absent the remaining two blocks. Thus, the experiment consisted of 2 stick conditions (present or absent) $\mathrm{x} 2$ ISI conditions (800 \& 80 or $80 \& 800)$. After all three taps, a white cylinder that projected outwards in space and was attached to the head of the participants (i.e., virtually linked to the HMDs) appeared and was used to point to the perceived location of each tap (see Figure 1C). Once the participants were pointing at the location they perceived the first tap, they used foot-pedal to log their response and proceed with the reporting the perceived location of the second and third taps in the same manner. All visual stimuli were presented via an HTC Vive head mounted display (HMD) equipped with a position tracking system. Stimulus presentation and data collection were controlled using Unity 3D Software (version 5.3.6f1) and custom scripts in C\#.

\subsection{Results and Discussion}

To examine whether the participants experienced an illusory sense of touch, the mean deviation of the participants' perceived location of each vibrotactile pulse from the actual location of the was of the vibrotactile stimulation was calculated. Because the pulse sequence went from left to right for half of the participants, and right to left for the other half of the participants, the data were reverse scored for the participants that received the stimuli from right to left, in order to make group comparisons. A 2 (Stick Present, Stick Absent) x 2 (CRI ISIs, non-CRI ISIs) repeated measures analysis of variance (ANOVA) was conducted to compare the mean deviation for each pulse (P1-P3). As expected, there were no significant main effects of stick presence $(\mathrm{F}(1,19)=0.79, \mathrm{p}=.38)$ or ISI condition $(\mathrm{F}(1,19)=0.94, \mathrm{p}=.344)$, and no significant interaction $(\mathrm{F}(1,19)=$ $1.21, \mathrm{p}=.28)$ for P1. Similarly, there was also no significant main effect of stick presence $(\mathrm{F}(1,19)=2.27, \mathrm{p}=.15)$ or ISI condition $(\mathrm{F}(1,19)=1.69, \mathrm{p}=.21)$, and no significant interaction $(\mathrm{F}(1,19)=0.09$, $\mathrm{p}=.77$ ) for P3. For P2, however, significant main effects of stick presence $(\mathrm{F}(1,19)=4.69, \mathrm{p}=.043)$, and ISI condition $(\mathrm{F}(1,19)=17.83$, $\mathrm{p}<.001)$ were observed, with no significant interaction between stick presence and ISI condition $(\mathrm{F}(1,19)=0.81, \mathrm{p}=.38)$. Planned comparisons between the mean deviations of the perceived location for the second tap and the first tap revealed an 'out of the body' cuntaneous rabbit illusion when the stick was both present $(\mathrm{t}(19)$ $=7.57, \mathrm{p}<.001, \mathrm{~d}=1.69,95 \%$ CI $[0.29,0.52])$ and absent $(\mathrm{t}(19)=$ $8.54, \mathrm{p}<.001, \mathrm{~d}=1.91,95 \% \mathrm{CI}[0.35,0.59])$ when the timing of the visuohaptic stimuli was consistent with the classic cutaneous rabbit illusion (see Figure 1D). Surprisingly, we also observed a significant out-of-the-body cutaneous rabbit in the conditions in which the visuohaptic stimuli were delivered using the non-cutaneous rabbit illusion timing, both when the virtual stick was present $(t(19)=$ $4.63, \mathrm{p}=.003, \mathrm{~d}=1.04,95 \% \mathrm{CI}[0.12,0.27])$, and when it was absent 
$(t(19)=5.44, p<.001, d=1.12,95 \%$ CI $[0.13,0.29])($ see Table 1 for additional analyses). P-values for all comparisons have been Bonferroni-corrected for multiple comparisons.

To examine whether the strength of the illusory sense of touch was stronger when the cutaneous rabbit ISIs were used compared to the non-cutaneous rabbit ISIs, the strength of the illusory sense of touch was calculated as the mean perceived deviation for P2 minus the perceived deviation of $\mathrm{P} 1$ for each condition. For the conditions in which the virtual stick was present, the illusory sense of touch was significantly stronger when the cutaneous rabbit timing was used compared to when the non-cutaneous rabbit timing was used $(\mathrm{t}(19)=4.05, \mathrm{p}=.012, \mathrm{~d}=.91,95 \% \mathrm{CI}[0.11,0.35])$. Similarly, the illusory sense of touch was significantly stronger when the cutaneous rabbit illusion timing was used compared to the noncutaneous rabbit illusion timing when the virtual stick was absent $(\mathrm{t}(19)=4.06, \mathrm{p}=.012, \mathrm{~d}=.91,95 \% \mathrm{CI}[0.12,0.38])$.

These results suggest that an illusory sense of touch was experienced in the space between the participants' hands when vibrotactile stimuli were delivered in a manner that was consistent with the CRI; both when a virtual stick was rendered between the participants hands and when no stick was rendered between the participants hands. The latter result suggests that we can experience a sense of touch, not only on virtual objects we are holding, but also in the empty space between our hands. These results also show that this illusion of touch was significantly reduced when the non-CRI ISIs were used, even though the visual stimuli were temporally congruent with the vibrotactile stimuli and presented in the same spatial locations as in the CRI ISI conditions. This suggests that the out-of-the-body illusory sense of touch cannot be explained by response bias or by changes in executive attention.

\section{EXPERIMENT 2}

In Experiment 2 we sought out to investigate whether the visual stimuli (i.e., white spheres) that appeared in the sequence consistent with the intended illusory percept in Experiment 1 was necessary to elicit the illusion. That is, we sought to investigate whether using the CRI ISIs of vibrotactile stimuli and the presence of a virtual stick would be sufficient to elicit the illusory sense of touch. Such a finding would be consistent with the original demonstration of the out-of-the-body CRI in which the out of the body illusory haptic percept was elicited by using the CRI ISIs for the vibrotactile stimuli when the participants were holding a ruler between their fingers, but was not elicited when the participants were not holding a ruler between their hands [Miyazaki et al. 2010]. However while the Miyazaki et al.[Miyazaki et al. 2010] out-of-the-body illusory touch required that the participants' eyes remained closed during the vibrotactile stimulation for the illusion to occur, here the participants' eyes would remain open. We hypothesized that, given the compelling nature of the visual virtual environment, the outof-the-body CRI would elicited when the vibrotactile stimuli were delivered using the CRI ISIs and a virtual object (i.e., the stick) was rendered between the participants' hands, but the illusion would not be elicited when there was no object (i.e., virtual stick) rendered between their hands.

\subsection{Method}

Twenty additional participants were recruited and participated in Experiment 2 (mean age $=27.75$ years, $\mathrm{SD}=4.99 ; 6$ females). In this experiment we sought to examine whether the illusory out-of-the-body CRI could be perceived without the visual stimuli (i.e., white spheres) presented in Experiment 1. Thus, only the CRI ISIs were used, and only the presence or absence of the stick was manipulated. The presence or absence of the stick, and the starting/ending location of and P1/P3 was counterbalanced across participants. All other materials and procedures were identical to Experiment 1.

\subsection{Results and Discussion}

A comparison between the mean deviation of the participants' reported perceived location of $\mathrm{P} 2(\mathrm{M}=0.06, \mathrm{SD}=0.06)$ compared to $\mathrm{P} 1(\mathrm{M}=0.03, \mathrm{SD}=0.05)$ revealed that there was a small but marginally significant out-of-the-body touch illusion when the stick was present $(\mathrm{t}(19)=3.04, \mathrm{p}=.05, \mathrm{~d}=.68,95 \% \mathrm{CI}[0.01,0.06])$ (see Figure 2). A single sample t-test revealed that the mean deviation of the perceived location of $\mathrm{P} 1$ was not significantly different from the actual site of stimulation (i.e., 0$)(\mathrm{t}(19)=2.53, \mathrm{p}=.17, \mathrm{~d}=.57,95 \%$ CI $[0.00,0.05])$. There was also no significant difference between the mean deviation of participants' location estimations for P2 compared to P3 $(\mathrm{M}=0.08, \mathrm{SD}=.07), \mathrm{t}(19)=-1.60, \mathrm{p}=1, \mathrm{~d}=.36,95 \%$ CI [-0.06, 0.01]. However, a significant difference between P3 and P1 was observed $(t(19)=1.60, p=1, d=.36,95 \% \mathrm{CI}[-0.06,0.01])$.

For the No Stick condition, a comparison between the mean deviation of the participants' reported perceived location of $\mathrm{P} 2(\mathrm{M}$ $=0.12, \mathrm{SD}=0.09)$ compared to $\mathrm{P} 1(\mathrm{M}=0.05, \mathrm{SD}=0.05)$ revealed illusion of touch $(\mathrm{t}(19)=3.20, \mathrm{p}=.04, \mathrm{~d}=.72,95 \% \mathrm{CI}[0.02,0.09])$. The mean deviation of $\mathrm{P} 1$ was also significantly different from the true location of the vibrotactile stimulation $(\mathrm{t}(19)=4.31, \mathrm{p}=.003, \mathrm{~d}$ $=.96,95 \% \mathrm{CI}[0.03,0.08])$.

The results from this experiment revealed that there was a very weak CRI when using the CRI ISIs, both when a stick was presented and when it was not. However, for both the Stick and No Stick conditions in this experiment, the mean perceived location of P2 was much closer to the true cite of stimulation than Experiment 1 , which suggests that this out-of-the-body illusion of touch was much weaker than when the additional synchronous visual cues were presented in the intended illusory location of $\mathrm{P} 2$.

\section{EXPERIMENT 3}

In Experiment 3 we sought to examine whether an illusory sense of touch could be elicited in an immersive VR setting using the tactile funneling illusion. The tactile funneling illusion is elicited when the amplitude of simultaneously presented vibrotactile stimuli presented at two disparate points are linearly modulated. The location of the illusory tactile percept depends on the amplitude difference of the vibrotactile stimuli [v. Békésy 1958]. Importantly, contrary to the cutaneous rabbit technique, this technique does not require a sequence of stimuli over time to elicit an illusion of touch, but rather can be elicited using one-shot simultaneous vibrotactile stimuli at two points. Here, we sought to examine whether (i) this illusion can be elicited on a virtual object in an immersive VR environment, and if so (ii.) whether visual stimuli presented simultaneously in the 


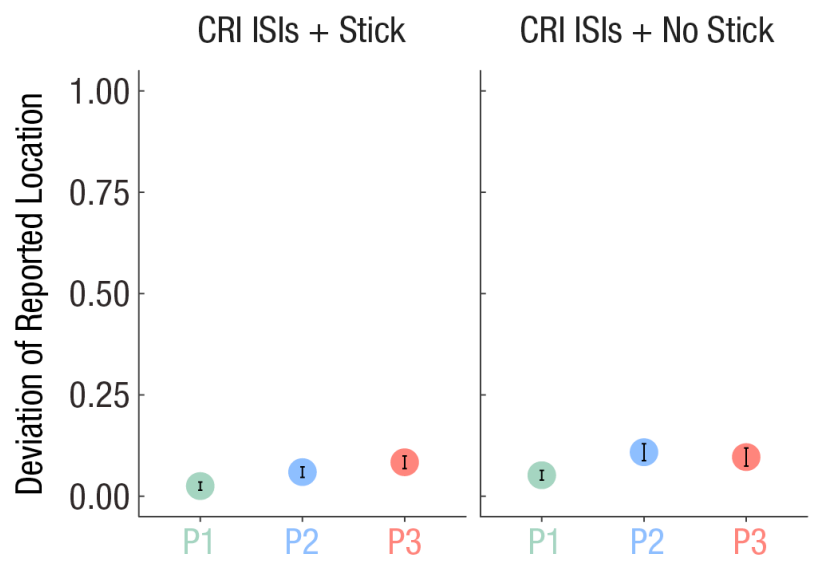

Figure 2: The Out-of-the-Body Touch Illusion in Experiment 2 when no additional visual cues (i.e., white spheres) were used. Mean deviation of perceived location for each pulse when the cutaneous rabbit illusion ISIs were used and when a virtual stick was either present (left) or absent (right). Error bars represent +/- 1 SEM.

intended illusory location are necessary to elicit the illusion. Thus, in Experiment 3, we employed the funneling technique to elicit the sense of touch in five different locations (P1-P5) in two conditions; one in which visual stimuli were simultaneously presented in the intended illusory location (Spheres Condition) and one in which they were not (No Spheres Condition). Following each vibrotactile pulse, the participants reported where they perceived the touch using a pointer extending outwards from their head in the virtual environment.

\subsection{Method}

4.1.1 Participants. Ten participants were recruited to participate in the experiment using the funneling technique (mean age $=31.4$ years, $\mathrm{SD}=7.04 ; 2$ females). All participants were recruited from within Microsoft Research, were healthy, reported no history of psychiatric illness or neurologic disorder, and reported no impairments of hearing or vision (or had corrected-to-normal vision).

4.1.2 Materials and Procedures. The participants received vibrotactile stimuli delivered simultaneously from two independent handheld Oculus Rift controllers while inside the same virtual environment as Experiments 1-2 and were holding a virtual stick that was rendered between their hands. Rift controllers were used for this experiment because such a modulation of the amplitude necessary for the funneling technique was not possible with the HTC Vive handheld controllers. The participants held the base of the controllers upside supported by their thumb and fingers with their palms facing upwards (as depicted in Figure 3A). The experiment consisted of two blocks 30 trials each. In one block, small white spheres (diameter $=18 \mathrm{~mm}$ ) were presented in the intended illusory location at the same time and for the same duration as the vibrotactile pulses (i.e., Spheres condition). In the other block no spheres were presented (i.e., No Spheres condition). Block order was counterbalanced across participants. For each trial, the vibrators contained in each handheld controller were set at one of five different amplitudes $(0, .25, .5, .75$ or 1.0$)$ and were activated briefly (100 ms) $2 \mathrm{~s}$ following the onset of the experiment or the previous trial. The linear modulation of the pulses was achieved by delivering simultaneous vibrotactile pulses of different amplitudes to the left and right controllers. The amplitudes combinations delivered to the left and right controllers were 1,$0 ; 0.75,0.25 ; 05,0.5 ; 0.25,0.75$; 0,1 , respectively, and corresponded to pulse amplitude modulations P1-P5 (see Figure 3B). Thus, each pulse amplitude modulation was intended to elicit the illusion that the vibrations were spatially located in one of 5 different locations (see Figure 3C). One of the five pulse amplitude modulations was delivered per trial, with each delivered in random order and repeated six times throughout the experiment. The desired amplitude and duration of vibrotactile pulses were set using custom scripts in C\# and implemented in Unity 3D Software (version 2017.1.0f3). This was done in accordance with previous research using the funneling technique to elicit illusory touch [Eimer et al. 2005; Richter et al. 2011]. After each pulse, a white cylinder that projected outwards in space and was attached to the head of the participants (i.e., virtually linked to the HMDs) appeared and was used to point to the perceived location of each of the vibration. Once the participants were pointing at the location they perceived the pulse, they used foot-pedal to log their response and proceed with next trial. All visual stimuli were presented via an Oculus Rift HMD equipped with the integrated 'Constellation' positional tracking system. The tracking system is enabled by stationary reference units that use optical IR LEDs and inertial sensors to track the user's head and handheld controllers. The Oculus Rift uses a Pentile OLED display with a 1080x1200 resolution per eye and a refresh rate of $90 \mathrm{~Hz}$. The effective field of view (FOV) for the participants is of 110 degrees.

\subsection{Results and Discussion}

A repeated measures analysis of variance revealed that there was a significant main effect of the amplitude modulation of vibrotactile stimuli on the perceived location of the touch for both the Spheres condition $(\mathrm{F}(1,9)=439.9, \mathrm{p}<.001)$ and the No-Spheres condition $(\mathrm{F}(1,9)=612.8, \mathrm{p}<.001)$. Subsequent planned comparisons were conducted to assess whether an illusory sense of touch was experienced on the stick in the intervening space between the hands for $\mathrm{P} 2-\mathrm{P} 4$ for the Spheres and No-spheres conditions. For the Spheres condition, a significant difference between the mean reported location of the sense of touch for $\mathrm{P} 2(\mathrm{M}=0.12, \mathrm{SD}=0.08)$ compared to $\mathrm{P} 1(\mathrm{M}=-0.01, \mathrm{SD}=0.04)(\mathrm{t}(9)=5.28, \mathrm{p}=.012, \mathrm{~d}=1.67,95 \% \mathrm{CI}$ $[0.07,0.18])$ and compared to $\mathrm{P} 5(\mathrm{M}=0.95, \mathrm{SD}=0.12)(\mathrm{t}(9)=-15.84$, $\mathrm{p}<.001, \mathrm{~d}=5.01,95 \% \mathrm{CI}[-.94,-.71])$. There was also a significant difference between the mean reported location of $\mathrm{P} 3(\mathrm{M}=0.45, \mathrm{SD}$ $=0.05)$ compared to $\mathrm{P} 1(\mathrm{t}(9)=34.11, \mathrm{p}<.001, \mathrm{~d}=10.79,95 \% \mathrm{CI}[0.42$, $0.49])$ and $\mathrm{P} 5(\mathrm{t}(9)=-15.50, \mathrm{p}<.001, \mathrm{~d}=4.90,95 \% \mathrm{CI}[-0.57,-0.42])$, and a significant difference between the mean reported location for $\mathrm{P} 4(\mathrm{M}=0.78, \mathrm{SD}=0.11)$ compared to $\mathrm{P} 1(\mathrm{t}(9)=20.44, \mathrm{p}<.001, \mathrm{~d}=$ $6.46,95 \% \mathrm{CI}[0.70,0.88])$ and P5 $(\mathrm{t}(9)=-6.61, \mathrm{p}=.002, \mathrm{~d}=4.90,95 \%$ CI $[-0.22,-0.12])$ (see Table 2 for additional comparisons).

Planned comparisons of the mean reported location of touch in the No-Spheres condition revealed that there was no significant 
Table 2: Mean localization comparisons for Experiment 3.

\begin{tabular}{|c|c|c|c|c|c|}
\hline Condition and Comparison & $t$ & $\mathrm{df}$ & $p$-value & $95 \% \mathrm{Cl}$ & Cohen's D \\
\hline \multicolumn{6}{|l|}{ Spheres Condition } \\
\hline P1 vs 0 & -0.50 & 9 & 1.00 & $-.04-.02$ & 0.16 \\
\hline$P 2$ vs $P 1$ & 5.28 & 9 & $.012^{*}$ & $.07-.18$ & 1.67 \\
\hline $\mathrm{P} 2$ vs $\mathrm{P} 3$ & -11.64 & 9 & $<.001^{* * *}$ & $-.40--.27$ & 3.68 \\
\hline P2 vs P5 & -15.84 & 9 & $<.001 * * *$ & $-.94--.71$ & 5.01 \\
\hline P3 vs P1 & 34.11 & 9 & $<.001^{* * *}$ & $.42-.49$ & 10.79 \\
\hline P3 vs P5 & -15.50 & 9 & $<.001^{* * *}$ & $-.57--.42$ & 4.90 \\
\hline P4 vs P1 & 20.44 & 9 & $<.001 * * *$ & $.70-.88$ & 6.46 \\
\hline P4 vs P3 & 10.02 & 9 & $<.001 * * *$ & $.26-.40$ & 3.17 \\
\hline P4 vs P5 & -6.61 & 9 & $.002^{* *}$ & $-.22--.12$ & 4.90 \\
\hline P5 vs 1 & -1.43 & 9 & 1.00 & $.86-1.03$ & 0.45 \\
\hline \multicolumn{6}{|l|}{ No Spheres Condition } \\
\hline $\mathrm{P} 1$ vs 0 & -0.75 & 9 & 1.00 & $-.03-.02$ & 0.24 \\
\hline $\mathrm{P} 2$ vs $\mathrm{P} 1$ & 2.72 & 9 & .596 & $.01-.17$ & 1.67 \\
\hline $\mathrm{P} 2$ vs $\mathrm{P} 3$ & -7.22 & 9 & $.001 * *$ & $-.46--.24$ & 2.28 \\
\hline P2 vs P5 & -16.52 & 9 & $<.001 * * *$ & $-.98--.74$ & 5.22 \\
\hline P3 vs P1 & 19.53 & 9 & $<.001 * * *$ & $.39-.49$ & 6.18 \\
\hline P3 vs P5 & -20.93 & 9 & $<.001 * * *$ & $-.57--.45$ & 6.62 \\
\hline P4 vs P1 & 22.78 & 9 & $<.001^{* * *}$ & $.77-.94$ & 7.20 \\
\hline P4 vs P3 & 13.60 & 9 & $<.001 * * *$ & $.35-.49$ & 4.30 \\
\hline P4 vs P5 & -4.58 & 9 & $.03 *$ & $-.14--.05$ & 1.45 \\
\hline P5 vs 1 & -3.61 & 9 & .141 & $.91-98$ & 1.14 \\
\hline \multicolumn{6}{|c|}{ Spheres Condition vs. No Spheres Condition } \\
\hline P1 & 0.21 & 9 & 1.00 & $-.02-.02$ & 0.07 \\
\hline P2 & 1.09 & 9 & 1.00 & $-.03-.12$ & 0.35 \\
\hline P3 & 0.70 & 9 & 1.00 & $-.04-.08$ & 0.22 \\
\hline P4 & -1.87 & 9 & 1.00 & $-.15-.01$ & 0.59 \\
\hline P5 & 0.11 & 9 & 1.00 & $-.06-.07$ & 0.04 \\
\hline
\end{tabular}

difference between $\mathrm{P} 2(\mathrm{M}=0.08, \mathrm{SD}=0.12)$ compared to $\mathrm{P} 1(\mathrm{M}$ $=-0.01, \mathrm{SD}=0.04)(\mathrm{t}(9)=2.72, \mathrm{p}=.596, \mathrm{~d}=1.67,95 \% \mathrm{CI}[0.01$, 0.17]). There was, however, a significant difference between the reported mean location for $\mathrm{P} 2$ compared to $\mathrm{P} 5(\mathrm{M}=0.94, \mathrm{SD}=0.05)$ $(t(9)=-16.52, p<.001, d=5.22,95 \%$ CI $[-0.98,-0.74])$. There was also a signficiant difference between in the mean reported location between P3 $(\mathrm{M}=0.43, \mathrm{SD}=0.07)$ compared to P1 $(\mathrm{t}(9)=19.53, \mathrm{p}<$ $.001, \mathrm{~d}=6.18,95 \% \mathrm{CI}[0.39,0.49])$ and $\mathrm{P} 5(\mathrm{t}(9)=-20.93, \mathrm{p}<.001, \mathrm{~d}=$ $6.62,95 \%$ CI $[-0.57,-0.45])$, and a signifcant difference in the mean reported location for $\mathrm{P} 4(\mathrm{M}=0.85, \mathrm{SD}=0.10)$ compared to $\mathrm{P} 1(\mathrm{t}(9)$ $=22.78, \mathrm{p}<.001, \mathrm{~d}=7.20,95 \% \mathrm{CI}[0.77,0.94])$ and $\mathrm{P} 5(\mathrm{t}(9)=-4.58, \mathrm{p}$ $=.03, \mathrm{~d}=1.45,95 \%$ CI $[-0.14,0.05])$.

Interestingly, there was no significant difference between the mean reported location of touch between the Spheres and NoSpheres conditions for P2 $(\mathrm{t}(9)=1.09, \mathrm{p}=1.00, \mathrm{~d}=0.35,95 \% \mathrm{CI}$ $[-0.03,0.12]), \mathrm{P} 3(\mathrm{t}(9)=0.70, \mathrm{p}=1.00, \mathrm{~d}=0.22,95 \% \mathrm{CI}[-0.04,0.08])$, or P4 $(\mathrm{t}(9)=-1.8, \mathrm{p}=1.00, \mathrm{~d}=0.59,95 \% \mathrm{CI}[-0.06,0.07])$ suggesting that, unlike in the CRI, the funneling illusion was not contingent upon visual cues in the location of the intended illusory touch percept. All p-values have been Bonferonni-corrected for multiple comparisons.

The results from this experiment show that an illusory sense of touch can be elicited at multiple points between the users' hands when using the tactile funneling technique. Moreover, the results from this experiment show that temporally congruent visual stimuli presented in the intended illusory location of the vibrotactile stimuli did not significantly strengthen the illusory out-of-the-body touch sensations.

\section{DISCUSSION}

In this study, we found that one's experience of touch can be extended beyond the body and onto virtual objects, and even into the empty space that surrounds us, by using virtual reality and two different vibrotactile stimulation techniques. The results from Experiment 1 revealed that an out-of-the-body CRI was experienced when the participants had their eyes open and viewed their virtual hands from a first person perspective. That is, our results show that the source of the second of three sequential vibrotactile pulses delivered to the hands via commercially available handheld controllers were perceived as being spatially located in the intervening space between the participants hands. We found that the out-of-the-body CRI was experienced when visual cues (i.e., white spheres) appeared at the same time and in the intended illusory location as the vibrotactile stimuli, even when there was no virtual stick rendered between the participants hands. The results from this study also showed that the visual cues were not sufficient to elicit the out-ofthe-body CRI as participants did not experience the illusion if the ISIs between vibrotactile stimuli was not consistent with the CRI. Specifically, the illusion was significantly reduced if the ISIs of the CRI were reversed, even when the visual cues were presented at the same time as each vibrotactile stimulus and in the intended illusory location. The results from Experiment 2, revealed that the out-ofthe-body CRI was not experienced if no synchronous visual cues 

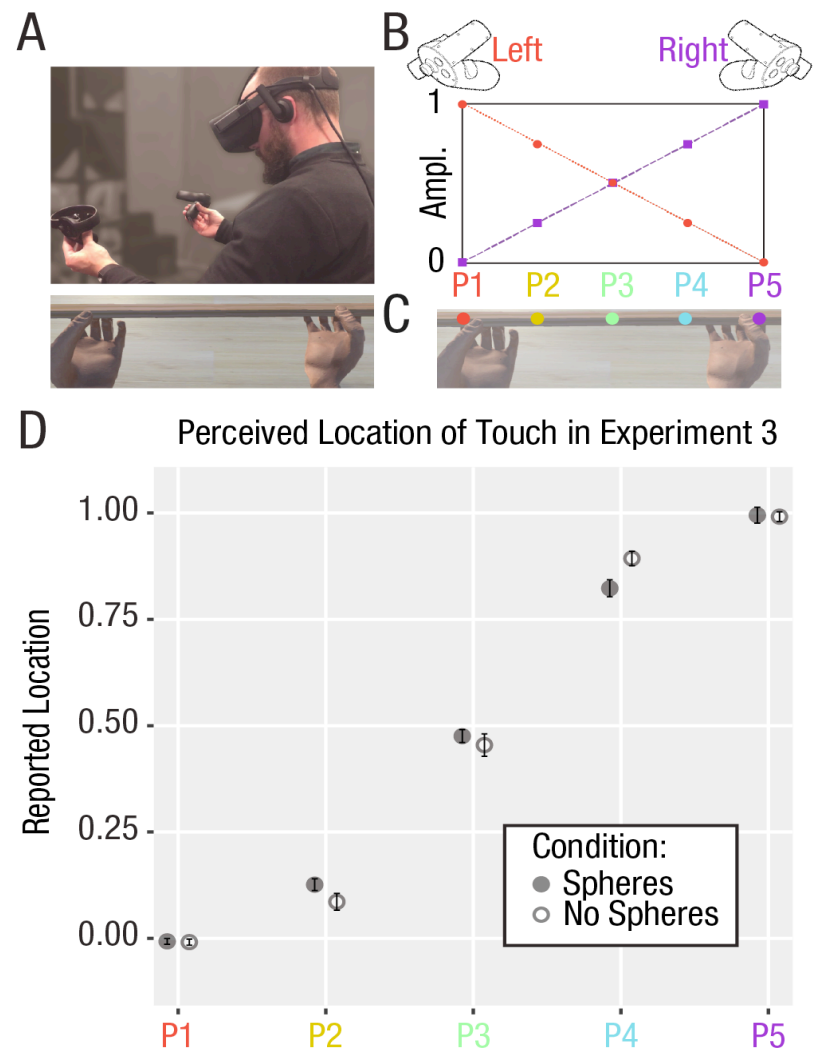

Figure 3: An Out-of-the-Body Touch Illusion from 'Funneling'. (A) The images depict the posture of the participants during the experiment (top), and the first person perspective of the hands as seen through he HMDs (bottom). (B) A schematic overview of the funneling technique by which linearly modulating the amplitude of vibrotactile stimuli simultaneously delivered to participants via the left and right Oculus Rift controllers elicits the illusory perception that a pulse occurs in the space between the hands. (C) The colored dots indicate participants' typical reported location for each amplitude modulation (P1-P5). (D) Mean reported location of each pulse type (P1-P5) for when additional visual stimuli (i.e., small white spheres) were also presented in the intended illusory location (Spheres Condition) and when no additional visual stimuli were presented (No Spheres Condition). Error bars represent +/- 1 SEM.

were presented, even when a virtual stick was rendered between the participants' hands. Combined, the results from Experiments 1 \& 2 suggest that, in virtual reality, synchronous visual cues presented in the intended illusory location of touch are necessary but not sufficient to elicit an out-of-the-body CRI between two handheld controllers when viewed from the first-person-perspective in an eye-open setting.

The results from Experiment 3 show that an illusory sense of touch can be experienced in the intervening space between the hands in an eyes-open setting in virtual reality, when simultaneous vibrotactile pulses are delivered to the left and right hand via handheld controllers. We found that by linearly modulating the amplitude between the two controllers, we could elicit an out-ofthe-body tactile funneling illusion of touch at three different points on a virtual object depending on the amplitude difference between the left and right controllers. Notably, unlike the out-of-the-body CRI, the out-of-the-body tactile funneling illusion was elicited both when visual cues were presented in the intended location, and when they were not. This finding was unexpected, and suggests that tactile funneling alone elicits a sufficiently robust illusion of spatialized touch that additional spatially and temporally congruent visual cues are not necessary to bolster the illusion. These findings may have important implications for future development of haptic devices, as it indicates that a robust illusion of touch can be elicited on virtual objects one is holding without the need for more, potentially distracting, visual cues in the environment. Our results also confer with recent work which has found that an illusion of tactile movement from one hand to the other can be elicited when holding two separate objects [Pittera et al. 2017]. Here, we demonstrate that an illusory sense of touch can be felt in the intervening space between the hands by merely manipulating the amplitude of the simultaneously presented stimuli and rendering a virtual object between them.

The results from Experiment $1 \& 2$ seem to contradict the findings of Miyazaki et al. [Miyazaki et al. 2010]. In the original demonstration of the out-of-the-body CRI Miyazaki et al. found that the illusion was only present when the participants had their eyes closed and believed that they were holding an object that was supported by their left and right fingertips (where the vibrotactile stimuli were delivered). If no ruler was present, the illusion was abolished. Thus, their results suggest that the top-down belief that a physical object connected the two points where the vibrotactile stimuli were in physical contact with the body was required for the illusion. By contrast, our findings show that the out-of-the-body CRI is experienced even when no virtual stick was rendered between the hands and they were completely independent. Moreover, Miyazaki et al. interpreted their findings within a theoretical framework by which our brain incorporates objects that we hold or manipulate into as part of our internal body schema [Yamamoto and Kitazawa 2001; Yamamoto et al. 2005]. However, our results show that sense of touch can be extended into the empty space that surrounds us, and suggests that a body-object interaction centered theory for the encoding of touch is incomplete. Future work may explore the neural underpinnings of this extracorporeal illusory sense of touch, in order to garner a more comprehensive understanding for how the brain represents our sense of touch in space.

The mis-localization of touch in external space observed in the present study is consistent with an amodal view of human perception, whereby, it is argued, the brain processes and integrates information from the senses in a content specific, rather than modality specific, manner [Proulx et al. 2014]. In this view, for example, the visual cortex is critically involved in processing spatial information, and the auditory cortex in critically involved in processing temporal information regardless of the modality from which that information is perceived [Maidenbaum et al. 2014; Poirier et al. 2007; Proulx et al. 2014; Striem-Amit et al. 2012]. This theory is supported by 
research on cross-modal plasticity and sensory substitution which has found, for example, that portions of the cortex previously believed to be responsible for vision alone are also activated in the blind when they perceive certain kinds of information delivered by sound, and that they can even learn to 'see' from sounds which contain spatial information about the environment [Proulx et al. 2014; Stiles and Shimojo 2015; Striem-Amit et al. 2012]. Future work on extra-corporeal touch illusions such as the ones observed in the present study will serve to examine the ways in which our sense of touch can be used as a proxy for delivering other kinds of sensory information.

Together with future work, the present findings will serve to inform design applications that involve delivering the sense of touch or delivering spatial information through touch from minimal points of physical contact with the skin. Given the predominance of controllers as the medium for which to interact with objects in current VR systems, this work will be immediately useful in creating more robust haptic experiences from controllers in VR. Specifically, by employing these haptic illusions in VR via controllers, users' sense of touch in VR can be expanded to virtual objects that appear in the intervening space between their hands, or that users wish to manipulate using two hands. This has the potential to make the make haptic experiences more congruent with the audiovisual environment in VR, which in turn will increase the sense realism and one's overall level of immersion in the virtual environment. These techniques for haptic rendering may also be applied to augmented reality settings, where from only two points of contact with the user (perhaps on each hand, or on two fingers on the same hand) vibrotactile stimuli can be used to generate haptic feedback from spatially diffuse virtual objects, buttons, or screens users interact with. Additionally, as progress continues to be made in the development of haptic suits for VR systems, the present findings may be used to allow developers to drastically reduce the number of stimulation sites while simultaneously maintaining a realistic and immersive haptic experience in VR.

\section{CONCLUSIONS}

In three separate experiments, using two different tactile illusions, we demonstrate that an illusory sense of touch can be felt in the intervening space between the hands when delivering vibrotactile stimuli to two independently handheld controllers in an immersive VR setting. These findings suggest that out sense of touch is not only limited to our body or even to physical objects we are holding, but rather can be expanded beyond, to the external space that surrounds us. The work presented here provides key insights into how we represent the sense of touch generally, as well as possible means with which to augment the human sense of touch, or the information delivered tactilely from multimodal devices, wearable devices, and in virtual reality settings in which there are limited points of physical contact with the user.

\section{REFERENCES}

Tomohisa Asai and Noriaki Kanayama. 2012. "Cutaneous rabbit" hops toward a light: Unimodal and cross-modal causality on the skin. Frontiers in Psychology 3, OCT (2012), 1-12. https://doi.org/10.3389/fpsyg.2012.00427

Felix Blankenburg, Christian C. Ruff, Ralf Deichmann, Geraint Rees, and Jon Driver. 2006. The cutaneous rabbit illusion affects human primary sensory cortex somatotopically. PLoS Biology 4, 3 (2006), 0459-0466. https://doi.org/10.1371/journal.pbio.
0040069

Gregory Burton. 1993. Non-neural extensions of haptic sensitivity. Ecological Psychology 5, 2 (1993), 105-124.

Li M. Chen, Robert M. Friedman, and Anna W. Roe. 2003. Optical Imaging of a Tactile Illusion in Area 3b of the Primary Somatosensory Cortex. Science 302, 5646 (2003), 881-885. https://doi.org/10.1126/science. 1087846

Martin Eimer, Bettina Forster, and Jonas Vibell. 2005. Cutaneous saltation within and across arms: A new measure of the saltation illusion in somatosensation. Perception \& Psychophysics 67, 3 (2005), 458-468.

Rudiger Flach and Patrick Haggard. 2006. The cutaneous rabbit revisited. fournal of Experimental Psychology: Human Perception and Performance 32, 3 (2006), 717-732. https://doi.org/10.1037/0096-1523.32.3.717

Frank A Geldard and Carl E Sherrick. 1972. The cutaneous "rabbit": A perceptual illusion. Science 178, 4057 (1972), 178-179.

Asif a Ghazanfar and Charles E Schroeder. 2006. Is neocortex essentially multisensory? Trends in cognitive sciences 10, 6 (Jun 2006), 278-285. https://doi.org/10.1016/j.tics. 2006.04.008

Eve Hoggan, Sohail Anwar, and Stephen A Brewster. 2007. Mobile multi-actuator tactile displays. In International Workshop on Haptic and Audio Interaction Design. Springer, 22-33.

Michael P. Kilgard and Michael M. Merzenich. 1995. Anticipated stimuli across skin. Nature 373, 6516 (1995), 663. https://doi.org/10.1038/373663a0

Jaedong Lee, Youngsun Kim, and Gerard J Kim. 2012. Funneling and Saltation Effects for Tactile Interaction with Virtual Objects. (2012), 3141-3148.

Shachar Maidenbaum, Sami Abboud, and Amir Amedi. 2014. Sensory substitution: Closing the gap between basic research and widespread practical visual rehabilitation. Neuroscience and Biobehavioral Reviews 41 (2014), 3-15. https: //doi.org/10.1016/j.neubiorev.2013.11.007

Makoto Miyazaki, Masaya Hirashima, and Daichi Nozaki. 2010. The "cutaneous rabbit" hopping out of the body. Fournal of Neuroscience 30, 5 (2010), 1856-1860.

Dario Pittera, Marianna Obrist, and Ali Israr. 2017. Hand-to-hand: an intermanual illusion of movement. Proceedings of the 19th ACM International Conference on Multimodal Interaction - ICMI 2017 (2017), 73-81. https://doi.org/10.1145/3136755. 3136777

Colline Poirier, Anne G. De Volder, and Christian Scheiber. 2007. What neuroimaging tells us about sensory substitution. Neuroscience and Biobehavioral Reviews 31, 7 (2007), 1064-1070. https://doi.org/10.1016/j.neubiorev.2007.05.010

Michael J. Proulx, David J. Brown, Achille Pasqualotto, and Peter Meijer. 2014. Multisensory perceptual learning and sensory substitution. Neuroscience and Biobehavioral Reviews 41 (2014), 16-25. https://doi.org/10.1016/j.neubiorev.2012.11.017

Hendrik Richter, Alina Hang, and Benedikt Blaha. 2011. The PhantomStation: Towards funneling remote tactile feedback on interactive surfaces. In Proceedings of the 2nd Augmented Human International Conference. ACM, 5.

Oliver S Schneider, Ali Israr, and Karon E MacLean. 2015. Tactile animation by direct manipulation of grid displays. In Proceedings of the 28th Annual ACM Symposium on User Interface Software \& Technology. ACM, 21-30.

Barry E Stein and Terrence R Stanford. 2008. Multisensory integration: current issues from the perspective of the single neuron. Nature reviews. Neuroscience 9, 4 (Apr 2008), 255-266. https://doi.org/10.1038/nrn2331

Noelle R B Stiles and Shinsuke Shimojo. 2015. Auditory Sensory Substitution is Intuitive and Automatic with Texture Stimuli. Scientific Reports 5 (2015), 15628. https://doi.org/10.1038/srep15628

Ella Striem-Amit, Laurent Cohen, Stanislas Dehaene, and Amir Amedi. 2012. Reading with Sounds: Sensory Substitution Selectively Activates the Visual Word Form Area in the Blind. Neuron 76, 3 (2012), 640-652. https://doi.org/10.1016/j.neuron.2012. 08.026

G v. Békésy. 1958. Funneling in the nervous system and its role in loudness and sensation intensity on the skin. The fournal of the Acoustical Society of America 30, 5 (1958), 399-412.

Jay P Warren, Marco Santello, and Stephen I Helms Tillery. 2010. Electrotactile stimuli delivered across fingertips inducing the Cutaneous Rabbit Effect. Experimental brain research 206, 4 (2010), 419-426.

Shinya Yamamoto and Shigeru Kitazawa. 2001. Sensation at the tips of invisible tools. Nature Neuroscience 4, 10 (2001), 979-980. https://doi.org/10.1038/nn721

Shinya Yamamoto, S Moizumi, and S Kitazawa. 2005. Referral of tactile sensation to the tips of L-shaped sticks. Fournal of Neurophysiology 93 (2005), 2856-2863. https://doi.org/10.1152/jn.01015.2004. 